\title{
Seroprevalencia y conocimiento de vacunación en nuevos residentes
}

\author{
Seroprevalence and vaccines knowledge \\ among newcomers hospital residents
}

\author{
Serrano Ramos, C. \\ Jiménez Bajo, L. \\ Bardón Fernández-Pacheco \\ Valle Robles, M. L. I. \\ O'Connor, S. \\ Caso Pita, C. \\ Servicio de Prevención de Riesgos Laborales \\ del Hospital Clínico San Carlos de Madrid. \\ Correspondencia: \\ Hospital Clínico San Carlos \\ Servicio de Prevención / Salud Laboral \\ c/Prof. Martín Lagos s/n \\ CP: 28040 Madrid
}

Resumen

Objetivo: Valorar el conocimiento de los nuevos residentes sobre su calendario vacunal, su estado serológico frente enfermedades vacunoprevenibles y la validez de la historia clínico-laboral como indicador de protección frente éstas.

Método: Mediante cuestionario específico obtenemos datos sobre el conocimiento del estado e historia vacunal, y mediante serología comprobamos el estado de protección actual de 130 nuevos residentes hospitalarios.

Resultados: La edad media es de 26,7 años, siendo $62 \%$ mujeres. Son españoles $82 \%$, de Centro y Sur América $17 \%$ y un $1 \%$ europeos. El $42 \%$ desconocen su calendario vacunal. Están protegidos frente varicela 93,1\% y frente a sarampión 90.8\%, pero frente parotiditis y rubéola sólo presentan anticuerpos positivos el $69.2 \%$ y $61.5 \%$ respectivamente. Refieren vacunación frente hepatitis B $81.5 \%$ y están protegidos $80,8 \%$. Refieren vacunación de hepatitis A 35\% y están protegidos 52.3\%. 65\% nunca se vacunó de gripe.

Conclusiones: Se ha objetivado un gran desconocimiento sobre vacunaciones. La protección frente parotiditis y rubéola ha resultado escasa. La cobertura vacunal frente a VHB, VHA y gripe es baja contando con que se trata ya de personal de riesgo antes de realizar este estudio. La historia clínico laboral no es congruente con los resultados serológicos en muchos casos.

Palabras Clave: Vacunación; Seroprevalencia; Residente bospitalario; Trabajador sanitario, Med Segur Trab 2009; 55 (214): 101-110

\section{Abstract}

Objective: To evaluate knowledge about vaccines, protection against vaccine-preventable diseases and labour medical history value.

Methods: We obtained data on immunization status and serum antibodies against these diseases from 130 newcomers residents to our hospital that were enrolled from May to June 2008. 
Results: Their mean age was 26.7 years (62\% women). They are Spanish $82 \%$, from Centre and South America $17 \%$ and 1\% European. 42\% of subjects don't know their immunization schedule. 93.1\% are immune against varicella and $90.8 \%$ against measles, but only $69,2 \%$ are immune against rubella and $61,5 \%$ against mumps. $81 \%$ have been vaccinated against hepatitis $\mathrm{B}$ and $80,8 \%$ are protected. $35 \%$ related to be vaccinated against hepatitis A, but $52.3 \%$ are protected. $65 \%$ of them never have been flu vaccinated.

Conclusion: A lack of knowledge about vaccine has been found. Protection against mumps and rubella is not enough. Immunization coverage against VHB, VHA and flu is low for this biological risk population. Lot of the subjects doesn't have a labour medical history consistent with serological results.

Key Words: Vaccination; Seroprevalence; Hospital Resident; Healthcare worker, Med Segur Trab 2009; 55 (214): 101-110 


\section{INTRODUCCIÓN}

El personal sanitario se ve afectado principalmente por el riesgo biológico, y por tanto los programas de inmunización entre estos trabajadores tiene una gran importancia para el control de este riesgo. Conocer la información del estado inmunológico del personal ante las enfermedades vacunoprevenibles es necesario para establecer medidas de protección ante estas enfermedades, permitiendo establecer protocolos de vacunación, aislamiento, protección individual o profilaxis tras exposición accidental, y evitando la infección de los trabajadores, sus familiares, sus compañeros y de los pacientes a los que tratan. Las vacunas recomendadas como prioritarias en el personal sanitario para los centros de control y prevención de enfermedades americano (Centres for Disease Control and Prevention, CDC) y el comité asesor de prácticas de inmunización (Advisory Comité on Immunization Practices, ACIP) son: paperas, rubéola, sarampión, varicela, gripe y hepatitis B como prioritarias, y Hepatitis A, Meningococo, Tos ferina, Fiebre tifoidea en segundo lugar, según otros factores ${ }^{1}$.

Los programas de vacunación infantiles reducen el desarrollo de estas enfermedades, y por tanto los adultos que no las padecieron en la niñez son susceptibles de enfermar en la edad adulta aunque fueran vacunados de las mismas, ya que la inmunogenicidad aportada por estas vacunas se reduce con el tiempo. Además dichas enfermedades, llamadas "propias de la infancia", se muestran en el adulto con mayor severidad y complicaciones que en la niñez ${ }^{2,3}$.

El personal sanitario, a pesar de tener un riesgo biológico diario en el trabajo, no tiene un conocimiento profundo sobre las vacunas y su importancia. Tampoco tiene conciencia real de peligro frente a estas enfermedades y en muchos casos no asume ninguna política de prevención hasta que en cierto modo se le exige que lo haga ${ }^{4,5,6}$. Menos aún los estudiantes del mundo sanitario, que con un riesgo biológico equiparable al de los trabajadores, presentan un perfil serológico similar al de la población general ${ }^{3,7}$.

Este estudio se centra en los residentes que generalmente, acaban de ser estudiantes para pasar a ser personal sanitario. Este grupo, puede ser representativo de otros colectivos del sector sanitario de edad similar, que comienzan su actividad laboral en el hospital. Así como de los estudiantes de todas estas profesiones que realizan prácticas hopitalarias.

A la hora de realizar una determinación de seroprevalencia previa a la incorporación del trabajo, se plantea la duda del coste-efectividad de la misma ante enfermedades posiblemente padecidas en la infancia que confieren inmunogenicidad de forma duradera $^{2}$. El servicio de prevención de riesgos laborales del Hospital Clínico San Carlos realiza la serología de Paperas, Rubéola, Sarampión, Varicela, Hepatitis B y Hepatitis A, a todos los residentes de nueva incorporación, y en el caso de especialidades intervencionistas también de VIH y VHC. Todas ellas son enfermedades frente a las cuales es imprescindible o muy recomendable que el personal sanitario se proteja según los organismos internacionales y sin embargo, la mayoría de los trabajadores no aportan documentación sobre su vacunación ni estado serológico frente a las mismas. A pesar de realizar historia clínica previa a la serología, y el hecho de inmunoprotección tras historia de enfermedad documentada mostrado por la mayoría de estudios $3,7,8,9,10$, dado el grado de imprecisión de la misma por ser enfermedades padecidas en la niñez, incluso en algunos casos de forma asintomática, y la posibilidad de no estar protegido frente a las mismas o de estarlo sin conocimiento, consideramos eficiente la serología prevacunación para todas ellas.

Los objetivos de este estudio son: describir el conocimiento de los residentes de nueva incorporación sobre su calendario vacunal y estado serológico, determinar seroprevalencia de anticuerpos frente: sarampión, rubéola, parotiditis, varicela, VHA y VHB, valorar la validez de la historia clínica como indicador de protección frente enfermedades vacunoprevenibles y describir la prevalencia de vacunación frente a la gripe. 


\section{MATERIAL Y MÉTODO}

\section{Diseño del estudio:}

Se ha diseñado un estudio observacional transversal, con un período de duración de cuatro meses (Mayo 2008- Septiembre 2008) realizado en el Hospital Clínico San Carlos. Se trata de un hospital universitario del área 7 de Madrid en el que trabajan unos 6000 profesionales, de entre ellos unos 600 médicos residentes.

\section{Población de estudio:}

Residentes incorporados al hospital Clínico San Carlos durante Mayo-Junio del 2008. Residentes de medicina (incluidos los de medicina familiar y comunitaria), residentes de psicología clínica, de farmacia clínica y biología clínica, que han sido incorporados durante el mismo período de tiempo, teniendo un total de 130 sujetos de estudio.

\section{Recogida de información:}

Se registran las variables mediante cuestionario específico diseñado para este estudio que consta de dos partes.

La primera es completada por el sujeto durante el reconocimiento médico inicial realizado por su incorporación al trabajo, y es revisada en conjunto con el investigador. Esta primera parte consta de las siguientes variables: Datos epidemiológicos (sexo, edad, lugar de nacimiento, especialidad y antecedentes laborales), conocimiento sobre el calendario vacunal infantil y calendario del adulto (conozco o no, vacunas administradas en el mismo), otras vacunas administradas y enfermedades padecidas. Además de recoger directamente los sujetos que reconocen no conocer su calendario vacunal infantil, se realizan unas preguntas sobre las vacunas infantiles que creen les han sido administradas, detectando así aquellos que aún creyendo conocer su calendario vacunal, no lo hacen.

El investigador tras el reconocimiento médico, completará la segunda parte del cuestionario en la que se recogen la serología pre-vacunación de VHB, VHA, varicela, parotiditis, sarampión y rubeola.

Todos los datos se registran en base informática Excel para su posterior análisis.

El estudio serológico se realiza tomando una muestra de sangre de todos los residentes de $5 \mathrm{ml}$ que es procesada para detectar anticuerpos Inmunoglobulina $\mathrm{G}$ específicos frente a paperas, rubéola, sarampión, varicela y hepatitis A, y anticuerpos frente al antígeno de superficie de hepatitis B (HBs) mediante la técnica de ELISA. Se considera a un sujeto inmune frente a hepatitis B si la presencia de anticuerpos frente HBs es mayor o igual a $10 \mathrm{IU} / 1$, y frente a hepatitis A si son mayores o igual a $20 \mathrm{IU} / 1$. En todos aquellos que no son inmunes frente a alguna de estas enfermedades, o no han completado la vacunación completa se les ofrece y recomienda la vacunación. Se realiza una nueva serología sólo en caso de requerir vacunación frente a la hepatitis al cabo de un mes tras completar vacunación. Sólo se realiza en estos casos serología post-vacunación para detectar no respondedores, debido a la gravedad de la enfermedad y porque la eficacia de la vacuna está entre el 80 y 95\%, habiendo una posibilidad de no respondedores. La seroconversión en caso de vacunación triple vírica se produce entre el 95-98\% con una eficacia del $99 \%$, y la efectividad de la vacuna contra varicela moderada o grave está entre el 97 y 100\% por lo que no se considera eficiente realizar serologías post-vacunales de manera general para estas enfermedades ${ }^{2}$.

\section{Análisis estadístico:}

Se realiza un análisis descriptivo de las principales variables. Para las variables cuantitativas empleamos medias para definir los resultados, con un intervalo de confianza al $95 \%$ y utilizamos frecuencias para las variables cualitativas.

Analizamos de manera general las variables de estudio y clasificamos según especialidad escogida (médica, quirúrgica, otras), según grupo etario y según trabajos 
anteriores para analizar el conocimiento sobre vacunación infantil, las vacunas administradas en la edad adulta y el estado serológico de los sujetos. Se realiza posteriormente tras estos resultados, un análisis estadístico Ji-cuadrado para ver la posible relación entre estas variables cualitativas.

También calculamos el valor predictivo positivo (VPP) y negativo (VPN) de la historia clínica para la varicela, las paperas, sarampión, VHB, VHA, tanto de historia de enfermedad como de vacunación referida.

Se ha utilizado el programa estadísticos SPSS versión 16.0

\section{RESULTADOS}

Desde finales de Mayo hasta finales de Septiembre de 2008, un total de 130 residentes fueron incluidos en este estudio. El 61\% son mujeres, con una edad media de 26,8 años (teniendo una moda de 25 , y con un intervalo de confianza para la media al $95 \%$ entre 26,13 y 27, 58). La distribución por lugar de nacimiento está representada en el gráfico 1.

Gráfico 1. Distribución por lugar de nacimiento.

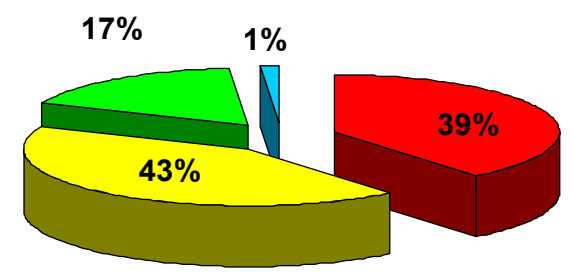

Españoles 81,5\%

Extranjeros $18,5 \%$

Madrid $\square$ Rest España $\square$ HispanoAmeric $\square$ Europa

Respecto a la distribución por especialidad escogida, el $53,8 \%$ va a realizar una especialidad médica clínica, el 23,1\% una quirúrgica, y un $19.3 \%$ otra especialidad, siendo el restante un 3,8\% de residentes de psicología, biología y farmacia clínica.

Para un $72 \%$ este es su primer empleo, del restante 28\%, la gran mayoría (89\%) trabajó en el sector sanitario. El porcentaje de haber tenido trabajos anteriores entre los mayores de 30 años es del 60\%, superior al de los menores de 30 años que es $23.5 \%$, habiendo una relación estadísticamente significativa causal entre estas dos variables (a mayor edad, es más probable haber trabajado anteriormente)

El $100 \%$ de los sujetos cree haber completado su calendario vacunal infantil, pero sólo el $58.5 \%$ de los sujetos lo conocen. Del $70 \%$ de los sujetos que dicen conocer su calendario vacunal y completan el cuestionario, un $16.5 \%$ comete errores (dice estar vacunados de viruela y/o no haber sido vacunados de polio, siendo nacido en España más tarde de 1982). Los porcentajes para conocimiento de vacunación según sexo, edad, antecedentes laborales y especialidad, son similares.

Los sujetos que refieren haber recibido alguna dosis vacunal, tanto infantil como del adulto son $81.5 \%$ frente a hepatitis $\mathrm{B}$, el $38.5 \%$ frente VHA, el $33.8 \%$ frente a la gripe y el recuerdo del adulto frente a tétanos difteria el $55.4 \%$. Además se han vacunado frente a: fiebre tifoidea el 19.2\%, fiebre amarilla el 20\%, cólera el 6.9\%, rabia el 4.6\%, neumococo el $1.5 \%$ y meningococo el $3.8 \%$, todos ellos por viajes internacionales.

HEPATITIS: Al comparar el estado de vacunación y la protección por antecedentes laborales, grupos de edad, especialidad, conocimiento de calendario vacunal infantil y nacionalidad, tanto en la vacunación frente a hepatitis B como a hepatitis A, se objetivan diferencias, pero sin una relación estadística significativa. (Ver tabla I) 
Tabla I. Prevalencia de vacunación y anticuerpos en títulos protectores de hepatitis B y hepatitis A.

\begin{tabular}{lcccc}
\hline & \multicolumn{2}{c}{ VHB } & \multicolumn{2}{c}{ VHA } \\
\cline { 2 - 5 } & VACUNACIÓN & PROTECCIÓN & VACUNACIÓN & PROTECCIÓN \\
\hline Trabajo & 84.4 & 84.4 & 37.5 & 59.4 \\
No trabajo & 80.6 & 79 & 36.2 & 52.2 \\
$<30$ a & 81.7 & 81.7 & 39.1 & 52.9 \\
$>30$ a & 80.0 & 73.3 & 13.3 & 40.0 \\
Quirúrgica & 90.0 & 83.3 & 30.0 & 53.6 \\
No quirúrgica & 80.0 & 60.0 & 44.3 & 53.0 \\
Conoce vacuna & 91.2 & 84.3 & 39.6 & 54.0 \\
No conoce & 59.0 & 53.8 & 28.2 & 51.0 \\
Español & 81.1 & 85.7 & 40.6 & 52.8 \\
Extranjero & 83.3 & 83.3 & 16.7 & 50.0 \\
Total & 81.5 & 80.8 & 39.2 & 53.1 \\
Adulto & 65.4 & 55.4 & 36.1 & 32.3 \\
Sólo Infantil & 15.4 & 13.8 & 6.9 & 5.3 \\
\hline & & & & \\
\hline
\end{tabular}

HEPATITIS B: Entre los sujetos que refieren vacunación completa en la edad adulta frente a hepatitis B hay un $15,3 \%$ que no está protegido (supuestos no respondedores) Del 39,2\% que refieren vacunación infantil, el 60,8\% está también vacunado en la edad adulta. Y entre los que sólo refieren vacunación infantil, hay un $10 \%$ que no está protegido, menor porcentaje que en la vacunación del adulto.

Hay un $4 \%$ que no refiere ninguna dosis vacunal, ni enfermedad pasada y aún así están protegidos frente hepatitis B. Ningún sujeto refirió enfermedad pasada.

HEPATITIS A: Entre los que dicen haber recibido vacunación completa, hay un $10.6 \%$ que no ha creado inmunidad. Y de los que refieren enfermedad pasada, también hay un $40 \%$ que no está inmune.

Todos los que refieren vacunación infantil son menores de 30 años, y de ellos el 55\% ha recibido vacuna del adulto. Un 3.8\% refieren enfermedad previa (todos ellos hispanoamericanos).

Entre los que tienen Ac. VHA en títulos protectores, hay un $29.5 \%$ que están protegidos, y no tienen antecedentes en la anamnesis que lo justifiquen. Éstos son $40 \%$ hispanoamericanos. Se podría suponer que han pasado enfermedad en la niñez o han recibido vacuna que no recuerdan.

VARICELA: El 93.1\% de los sujetos de la muestra están protegidos frente a varicela, sin variar el porcentaje significativamente al comparar por grupos de edad, nacimiento o antecedentes laborales. Entre el $\mathbf{8 4 . 6 \%}$ que refiere paso de enfermedad, hay dos sujetos que no presentan anticuerpos protectores. Refieren haber sido vacunados dos sujetos, de los cuales sólo uno está protegido. (Tabla II)

TRIPLE VÍRICA: Refieren no tener administrada la vacunación triple vírica de la infancia ocho sujetos de la muestra. Entre ellos, cuatro son extranjeros y dos mayores de 30 años. Todas las diferencias halladas, no tienen significación estadística. (Tabla III)

PAROTIDITIS: Al comparar la positividad de anticuerpos frente a parotiditis por grupos de edad, nacimiento o antecedentes laborales, es superior en españoles y en menores de 30 años. El porcentaje que refieren haber padecido enfermedad, es mayor entre los extranjeros, que en la población española. De los que refieren haber pasado 
enfermedad, están protegidos el 85\%. VPP de historia de enfermedad es bajo, y el VPN es alto en la muestra, probablemente debido a la baja prevalencia de enfermedad.

SARAMPIÓN: De los que refieren haber padecido enfermedad, está protegido el 94.7\%. El porcentaje es mayor de haber padecido enfermedad entre los mayores de 30 años, y respecto a la nacionalidad es muy similar.

RUBEOLA: Entre los que refieren paso de enfermedad, el 75\% presenta títulos de anticuerpos positivos. Al comparar el antecedente de enfermedad padecida por grupos de edad y nacionalidad, tienen un porcentaje mayor los mayores de treinta años y los extranjeros.

GRIPE: Ninguna de las diferencias encontradas, son estadísticamente significativas, ni respecto a la actividad laboral previa, ni respecto a la edad. A pesar de esto, es importante destacar la mayor prevalencia de vacunación entre los que no han trabajado en el mundo sanitario, y los que mayor conocimiento tienen sobre vacunación.

Tabla II. Antecedentes referidos de paso de enfermedad (Ant.Enf.), anticuerpos positivos (Acs.Posit.), y valores predictivos positivo (VPP) y negativo (VPN)

\begin{tabular}{|c|c|c|c|c|c|c|c|c|}
\hline & & TOTAL & $\begin{array}{c}\text { Menor } \\
<\mathbf{3 0}\end{array}$ & $\begin{array}{c}\text { Mayor } \\
>\mathbf{3 0}\end{array}$ & Español & Extranjero & VPP & VPN \\
\hline \multirow{2}{*}{ VARICELA } & Ant. Enf. & 84.6 & 85.2 & 80 & 85.8 & 79.2 & \multirow{2}{*}{89.3} & \multirow{2}{*}{87.5} \\
\hline & Acs. Posit. & 93.1 & 93 & 93.3 & 94.3 & 87.5 & & \\
\hline \multirow{2}{*}{ SARAMPIÓN } & Ant. Enf. & 14.6 & 13 & 26.7 & 15.1 & 12.5 & \multirow{2}{*}{15} & \multirow{2}{*}{90} \\
\hline & Acs. Posit. & 90.8 & 91.3 & 86.7 & 91.5 & 87.5 & & \\
\hline \multirow{2}{*}{ RUBEOLA } & Ant. Enf. & 7.7 & 6.1 & 20 & 5.7 & 16.7 & \multirow{2}{*}{11} & \multirow{2}{*}{97} \\
\hline & Acs. Posit. & 61.5 & 61.7 & 60 & 60.4 & 66.7 & & \\
\hline \multirow{2}{*}{ PAROTIDITIS } & Ant. Enf. & 15.4 & 14 & 28.6 & 12.4 & 30.4 & \multirow{2}{*}{19} & \multirow{2}{*}{97} \\
\hline & Acs. Posit. & 69.2 & 70.4 & 60 & 70.8 & 62.5 & & \\
\hline \multirow{3}{*}{ VHA } & Ant. Enf. & 3.8 & 2.6 & 13.3 & 0.9 & 16.7 & \multirow{3}{*}{4.4} & \multirow{3}{*}{96.6} \\
\hline & Acs. Posit. & 52.3 & 54.9 & 40 & 53.8 & 50 & & \\
\hline & Vacun & 39.2 & 39.1 & 13.3 & 40.6 & 16.7 & & \\
\hline
\end{tabular}

Tabla III. Antecedente de vacunación de Gripe.

\begin{tabular}{|c|c|}
\hline GRIPE & \% Vacunados alguna vez \\
\hline 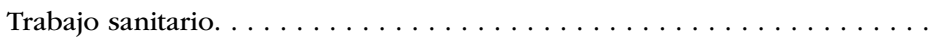 & 28.1 \\
\hline 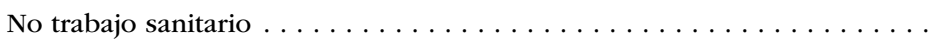 & 35.7 \\
\hline 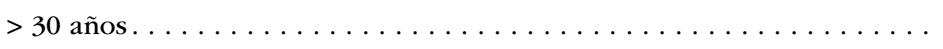 & 53.3 \\
\hline 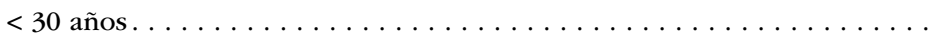 & 31.3 \\
\hline 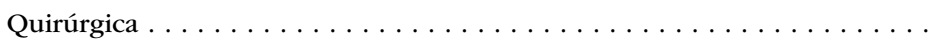 & 46.7 \\
\hline 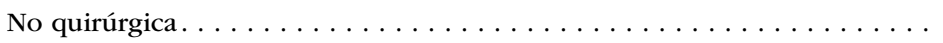 & 30.0 \\
\hline 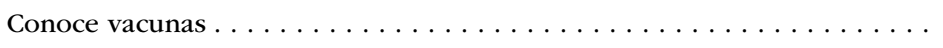 & 39.6 \\
\hline 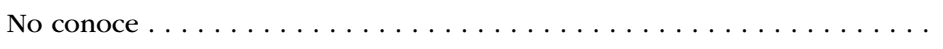 & 20.5 \\
\hline 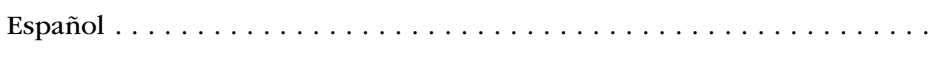 & 36.8 \\
\hline 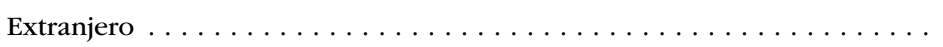 & 20.8 \\
\hline
\end{tabular}




\section{CONCLUSIONES}

La muestra escogida puede ser una buena representación de la población sanitaria que comienza a trabajar en los hospitales de Madrid. Mayoritariamente mujeres, jóvenes, españolas, aunque con un sector importante de trabajadores sanitarios de nacionalidad hispanoamericana.

El porcentaje del personal sanitario que tiene su calendario vacunal al día, incluso aún sin considerarlos personal de riesgo biológico, sino como población general, es muy bajo, sólo un $55 \%$ se puso la dosis de recuerdo frente a tétanos-difteria. De manera que como ya se ha objetivado anteriormente en la bibliografía, los programas de vacunación del adulto en España no han alcanzado el desarrollo de los infantiles, por lo que la vacunación en el medio laboral es de gran importancia, más aún si hablamos del sector sanitario $^{1,2}$

Se ha objetivado un gran desconocimiento sobre vacunaciones, a pesar de tratarse de un colectivo cuya sensibilización debería ser mayor que en la población general, sólo un $59 \%$ ha sido capaz de completar el sencillo cuestionario vacunal. Este desconocimiento sobre las vacunaciones, también se ha visto en varios estudios ${ }^{4,5,6,11}$, y estamos de acuerdo con los mismos en que una mayor formación respecto a la prevención con vacunación es necesaria en el personal sanitario. De este modo, es probable que los trabajadores de este sector tuvieran una mayor percepción del riesgo, un menor temor a los efectos adversos de la vacunación y una mayor confianza en los beneficios que aportan las vacunas en este colectivo, tanto evitando la propia enfermedad del trabajador como de los pacientes a los que trata. Además según los resultados obtenidos, parece que los sujetos que no fallaban el cuestionario vacunal, es decir que tienen mayor conocimiento sobre vacunas, tenían un mayor porcentaje de vacunados. Estas diferencias no han sido relevantes estadísticamente hablando, pero hay que tener en cuenta que la muestra es escasa para ello.

En todas las enfermedades estudiadas, el porcentaje tanto del estado de protección como de vacunación frente a las mismas es similar entre los que habían trabajado anteriormente como sanitarios como en los que no. Este dato nos debe hacer pensar en una Vigilancia de la Salud de estos trabajadores sanitarios deficiente. Es posible que este hallazgo estuviera sesgado, dado que los empleos anteriores como sanitarios en la mayoría de los sujetos de esta muestra, fueran de corta duración, y también porque la muestra está compuesta por sujetos pertenecientes al mundo sanitario como estudiantes, por lo que el porcentaje de vacunados es mayor que en población general. Pero aún así, debemos pensar en hacer un mayor esfuerzo en concienciar a los trabajadores sanitarios en los riesgos que corren, y mejorar la prevención de riesgos laborales.

Respecto a la hepatitis $\mathrm{B}$, hay incongruencia de la historia frente a la serología en un $7 \%$ de los casos, ya que tienen anticuerpos HBs en títulos protectores, y no refiere ninguna dosis vacunal ni historia sugerente de contacto con virus hepatitis B. Entre los que refieren vacunación completa frente a hepatitis $\mathrm{B}$, un $13,2 \%$ no está protegido, pero en este caso es posible que no hayan respondido a la primera pauta vacunal, que haya pasado demasiado tiempo desde la vacunación, que sean no respondedores o que no hayan recibido las tres dosis adecuadamente.

La prevalencia de hepatitis A en nuestro estudio parece mayor en países hispano americanos, ya que de los sujetos hispanoamericanos de esta muestra, 18\% refieren paso de enfermedad y otro $32 \%$ tienen niveles de Ac. VHA protectores sin antecedente vacunal, probablemente por haberla padecido en la niñez, teniendo así un $50 \%$ que podría haber padecido enfermedad. En los sujetos de nacionalidad Española sólo 1 persona refiere paso de enfermedad y hay 13 sujetos protegidos que no podemos explicar, teniendo un 13\% como máximo de prevalencia de hepatitis A. Estos resultados son congruentes con los datos sobre prevalencia de la hepatitis A en cuanto a que la prevalencia es mayor en países de Centro y Sur América que en España. 
La varicela parece que continúa siendo una enfermedad prevalente, con un 93\% de serologías positivas frente a la misma con un solo sujeto vacunado. Esta prevalencia es menor respecto al $\mathbf{9 5 , 1 \%}$ y $\mathbf{9 8 , 8} \%$ hallados en estudios con una población similar a la nuestra realizados en $2001^{3,10}$ En nuestra muestra, un $65 \%$ de los casos que no referían contacto con virus varicela zoster, estaban protegidos, otros estudios presentan mayores porcentajes $^{3,10}$. Hay también dos casos que referían haber padecido enfermedad, y sin embargo presentan anticuerpos negativos.

Más llamativa es la diferencia en la prevalencia de anticuerpos positivos frente a rubéola respecto al mismo estudio del Dr. Solano realizado en 2001. Pasando de un 98,3\% en 2001 a un 61,5\% en nuestra muestra. Frente a la parotiditis, sólo el 69,2\% tiene anticuerpos positivos, y sólo refiere paso de enfermedad el 15,4\%. De éstos que refieren enfermedad pasada, el 15\% no están protegidos.

En el caso del sarampión, aunque el antecedente de paso de enfermedad sólo lo refiere el 15,3\% de la muestra, hay un 90,3\% que presenta títulos positivos, probablemente por la vacunación triple vírica. Hay también un 5,3\% que refieren paso de enfermedad y no están protegidos.

En la muestra hay una baja tasa de protección frente al virus de rubéola y parotiditis, y aunque en menor medida un porcentaje considerable tampoco está protegido frente sarampión. Todo esto resalta la necesidad de revacunación en este grupo de edad frente las mismas, ya que cualquiera de ellas en el adulto, puede conllevar consecuencias graves.

Se ha objetivado incongruencia de la historia clínico-laboral respecto a los resultados serológicos frente a todas las enfermedades estudiadas. Hallamos muchos casos que no refieren paso de enfermedad y sin embargo están protegidos, así como algún caso que refiere haber padecido enfermedad o haber sido vacunado, y sin embargo, no tiene anticuerpos positivos frente a la misma. Esta incongruencia de la historia con los resultados serológicos, nos afirma la conveniencia de realizar serología prevacunal a todo el personal sanitario, como única medio para garantizar la protección del trabajador frente estas enfermedades.

La cobertura vacunal frente a virus de la gripe, es escasísima en este grupo de riesgo como es el personal sanitario. El porcentaje de vacunados de la muestra es muy bajo, pero además, hay que tener en cuenta que no es vacunación anual, sino vacunado alguna vez en la vida. La vacuna antigripal es la peor aceptada entre el personal sanitario a pesar de la indicación sistemática en el mismo $^{12}$, y como vemos en nuestro estudio, la cobertura de vacunación de gripe es la menor de todas las indicadas en el ámbito laboral sanitario.

Para mejorar la cobertura vacunal y por tanto la protección frente a las enfermedades vacunoprevenibles entre los trabajadores sanitarios, se deben implantar campañas de vacunación activas específicas, que conciencien sobre el riesgo, informen sobre los beneficios de la vacunación, y disminuyan el temor frente los efectos adversos posibles.

\section{BIBLIOGRAFÍA}

1. Centers for Disease Control and Prevention. Immunization of Health-Care Workers: recommendations of the Advisory Committee on Immunization Practices (ACIP) and the Hospital Infection Control Practices Advisory Committee (HICPAC). MMWR 1997;46(No. RR-18).

2. C.Caso Pita, F.Cruzet Fernández, J. de la Concepción Lucas, D. Insausti Macarrón, L.Rodríguez de la Pinta. Vacunación en el ámbito laboral. 2006. 6: 79-152.

3. MA. Villasís-Keever, L.A.Peña, G.Miranda-Novales, T.Alvarez y Muñoz, L.Damasio-Santana, G.López-Fuentes, J.L.Girón-Carrillo. Prevalence of Serological Markers against Measles, Rubella, Varicella, Hepatitis C, and Human Inmunodeficiency Virus among Medical Residentes in Mexico. Preventive Medicine 2001; 32: 424-428 
4. Esposito S, Tremolati E, Bellasio M, Chiarelli G, Marchisio P, Tiso B, Mosca F, Pardi G, Principi N; V.I.P. Study Group. Attitudes and knowledge regarding influenza vaccination among hospital health workers caring for women and children. Vaccine. 2007 Jul 20;25(29):5283-5289. Epub 2007 Jun 4.

5. Goins WP, Schaffner W, Edwards KM, Talbot TR. Healthcare workers' knowledge and attitudes about pertussis and pertussis vaccination. Infect Control Hosp Epidemiol. 2007 Nov;28(11):1284-1289. Epub 2007 Aug 27.

6. Wortley PM, Schwartz B, Levy PS, Quick L, Evans B, Burke B. Healthcare workers who elected not to receive smallpox vaccination. Am J Prev Med. March 2006; 30 (3) 258-265.

7. G. Baer, J. Bonhoeffer. U.B. Schaad, U.Heininger. Seroprevalence an immunization history of selected vaccine preventable diseases in medical students. Vaccine 23 (2005) 2016-2020

8. Celikbas A, Ergonul O, Aksaray S, Tuygun N, Esener H, Tanir G, Eren S, Baykam N, Guvener E, Dokuzoguz B. Measles, Rubella, mumps and varicella seroprevalence among health care workers in Turkey: Is prevaccination screenin cost-effective? Am J Infect Control. 2006 Nov;34(9):583-587

9. Vandersmissen G, Moens G, Vranckx R, de Schryver A, Jacques P. Occupational risk of infection by varicella zoster virus in Belgian healthcare workers: a seroprevalence study. Occup Environ Med. 2000 Sep;57(9):621626.

10.-V.M.Solano, M.J.Hernández, J.I.Martín, E.V.Martínez, A.Misiego, J.L.Arribas. Seroprevalencia de enfermedades vacunoprevenibles en médicos residentes. Medicina Preventiva 2006; 23(2): 11-16.

11. Wortley PM, Levy PS, Quick L, Shoemaker TR, Dahlke MA, Evans B, Burke B, Schwartz B. Predictors of smallpox vaccination among healthcare workers and other first responders. Am J Prev Med. 2007 Jun;32(6):538-541.

12. M. Campins Martí, J. M. Elorza Ricart, X.Martínez Gómez, A. Allepuz Palau, E.Ferrer Gramunt y M. MéndezAguirre Guitián. Vacuna antigripal y personal sanitario: estrategias para aumentar las coberturas en un hospital de tercer nivel. Med Clin (Barc) 2002;119(12):451-452

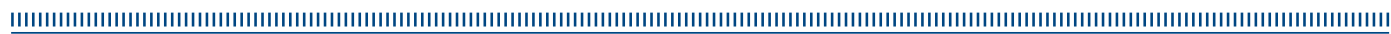

